\title{
WORKLOAD, JOB SATISFACTION AND OCCUPATIONAL STRESS IN POLISH MIDWIVES BEFORE AND DURING THE COVID-19 PANDEMIC
}

\author{
Arkadiusz Mirosław Jasiński, Romuald Derbis, Radosław Walczak \\ University of Opole, Opole, Poland \\ Institute of Psychology, Department of General and Work Psychology
}

\begin{abstract}
Background: The aim of this study was to describe, explain, and compare the correlations between workload, job satisfaction, and occupational stress levels in Polish midwives working before and during the COVID-19 pandemic. Material and Methods: This study was cross-lagged in nature and conducted in 2 phases at public health facilities. The first phase took place between March and August 2018 before the current pandemic, and the second in February 2021. In total, 133 midwives working before the COVID-19 pandemic and 92 midwives working during the pandemic were surveyed. Results: Analysis revealed that working during the pandemic, personal SARS-CoV-2 infection and workload were positive predictors of occupational stress. Midwives working during the pandemic experienced significantly higher levels of occupational stress compared to the period before the pandemic. Job satisfaction was found to be a negative predictor of stress in both groups, and to mediate the correlation between workload and occupational stress. The positive influence of job satisfaction on coping with stress was stronger in the group working during the pandemic. Conclusions: Workload increased, and job satisfaction decreased, occupational stress levels in both groups of midwives. The COVID-19 pandemic, directly and indirectly, increased midwives' occupational stress levels, and reinforced the negative correlation between workload and job satisfaction. The results confirm the important role of working conditions in shaping occupational stress levels. Med Pr. 2021;72(6):623-32
\end{abstract}

Key words: job satisfaction, midwives, occupational stress, COVID-19, mediation, JD-R model

Corresponding author: Arkadiusz Mirosław Jasiński, University of Opole, Institute of Psychology, Department of General and Work Psychology, pl. Staszica 1, 45-052 Opole, Poland, e-mail: arkadiusz.jasinski@uni.opole.pl

Received: May 25, 2021, accepted: September 2, 2021

\section{INTRODUCTION}

The first case of COVID-19 in Poland was diagnosed on March 4, 2020. Since then, more than 70000 people have died from the disease in this country, accounting for $2 \%$ of all infected people in the Polish population [1]. One of the most important areas of research in the context of COVID-19 is the psychological response to the pandemic. Medical professionals, compared to workers in other industries, are significantly more likely to be exposed to infected individuals. Healthcare workers also experience increased negative psychological symptoms in relation to work, including anxiety, insomnia and lower levels of psychological well-being [2]. Midwives represent one of the key professional groups in the healthcare sector. The quality of their work is a fundamental predictor of women's satisfaction with childbirth and the health outcomes of newborns [3]. Midwifery is a profession with high exposure to stress, and it is considered as one of the most physically and psychologically demanding professions. Research indicates that working during the pandemic is positively correlated with increased anxiety and uncertainty, and is negatively correlated with life satisfaction in this group [4].

This study was conducted based on the Job-Demands Resources (JD-R) model [5]. According to this theory, chronic stress is a major predictor of work-related burnout. High levels of workload and occupational stress, and low job satisfaction, can lead to a lower quality of health services. Therefore, the determinants of high levels of occupational stress in midwives should be evaluated.

The main objective of this study was to examine the correlations between workload, job satisfaction, and occupational stress levels in midwives working before and during the COVID-19 pandemic. To this end, the aims were: 1 ) to evaluate whether working under pandemic conditions positively correlated with the midwives' occupational stress levels and if 
midwives working during a pandemic experienced higher levels of occupational stress compared to those working before the pandemic; 2) to test whether personal COVID-19 infection was a positive predictor of occupational stress; 3 ) to describe and compare the strength of the correlations between workload, job satisfaction, and occupational stress in the 2 groups of midwives. The specific hypotheses tested in this study are outlined below.

\section{Working in pandemic conditions as a stressor}

The COVID-19 pandemic acts as a significant stressor that can deteriorate mental health, manifested by a range of symptoms including depression [6]. Psychologically, dealing with the pandemic may lead to rumination about COVID-19 [7]. A rumination-based coping strategy is ineffective as ruminations involve creating a mental representation of a negative event, leading to shortterm or prolonged stress reactions [8]. Previous studies have shown that, due to the pandemic, midwives were forced to change the organization of their work, which led to concerns about how those actions would affect the quality of healthcare [9]. Based on the results cited above, it was assumed that midwives' work during the pandemic would be positively correlated with occupational stress levels. Thus, the following hypotheses were outlined:

H1: midwives' work during the COVID-19 pandemic is positively correlated with occupational stress;

- H2: working as a midwife during the COVID-19 pandemic is more stressful compared to the period prior to the pandemic.

Kniffin et al. [10], in a comprehensive review of research on the possible psychological consequences of work in the context of the pandemic, highlighted the need for research on how personal SARS-CoV-2 infection affects employees. Previous studies have indicated that 2 out of 10 healthcare workers who have suffered from COVID-19 exhibit the symptoms of PTSD [11]. Hence, the decision was made to investigate how SARS-CoV-2 infection is related to the level of stress experienced at work. Therefore, the following hypothesis was outlined:

H3: personal SARS-CoV-2 infection in midwives is positively associated with occupational stress.

\section{Workload and occupational stress: the mediating role of job satisfaction}

According to the JD-R model, 2 dimensions of the work environment mediate occupational stress: requirements and resources [5]. Requirements are the physical, mental, and social demands needed to complete a job, while resources are those aspects of work that enable an individual to successfully complete the job and reduce the associated stress. According to the JD-R model, the requirements of work are responsible for shaping the mental health and well-being of employees, and this influence is moderated by the resources of the employee and the organization. Increased job requirements and low levels of resources can lead to job strain, chronic stress and, ultimately, a work-related burnout [5]. Workload is one of the basic requirements of work, and is increased by working under onerous pressure, including having to do a lot of work with insufficient time [12]. High levels of workload are a positive predictor of depressed mood and emotional exhaustion [13]. Midwives, due to the nature of their profession, very often perform their duties under time pressure. Based on the results cited above, it was hypothesized that having to quickly and correctly complete numerous tasks with high levels of responsibility may be positively correlated with occupational stress. Based on this assumption, the following hypothesis was proposed:

- H4: workload is positively associated with occupational stress in both study groups of midwives.

Job satisfaction is defined as the cognitive aspect of well-being at work. According to the JD-R model, the level of job satisfaction is a consequence of the available resources [14]. Job satisfaction among midwives may stem from the perception of their work as being socially important, including the perception of work conditions, prestige, accomplishments, and its potential for self-actualization. Previous studies have shown that employees who are satisfied with their jobs perform their duties more effectively, and the level of job satisfaction is positively correlated with the financial state of the organization [15]. According to the JD-R model, the level of job satisfaction can predict the level of occupational stress, an idea supported by previous work [16]. In midwives, cognitive job evaluation scores are likely to be related to the levels of occupational stress. Thus, the following hypothesis was formulated:

- H5: job satisfaction is negatively associated with occupational stress in both study groups of midwives.

If job satisfaction is correlated with both workload and occupational stress, it seemed rational to test whether the workload is related to occupational stress with job satisfaction acting as a mediator. Such an analysis is supported by the JD-R model, which suggests that the positive correlation between job requirements and the psychological costs of work is not necessarily straightforward, but is due to the presence of mediating 
variables. A mediator explains all or part of the effect of an independent variable on a dependent variable and mediation analysis allows one to identify and estimate the size of this effect [17]. It can be assumed that the correlation between workload and occupational stress will be weaker when job satisfaction is included as a mediator. Job satisfaction as a positive cognitive appraisal of working conditions can buffer the association between the time pressure, multiple responsibilities, and occupational stress. It was predicted that the strength of the association between the study variables would be significantly higher in the group of midwives working during the pandemic, compared to those working in the period before the pandemic. It was suspected that the pandemic context would intensify the positive correlation between workload and occupational stress, reinforce the negative effect of workload on job satisfaction, and increase the positive role of job satisfaction in coping with stress. To evaluate the assumptions listed above, the following hypotheses were proposed:

H6: job satisfaction mediates the correlation between workload and occupational stress;

H7: the strength of the associations between workload, job satisfaction, and occupational stress will be significantly higher among midwives working during the COVID-19 pandemic compared to those working before the pandemic.

\section{MATERIAL AND METHODS}

This study was questionnaire-based and cross-lagged in nature. The study design and presentation of results follow the Strengthening the Reporting of Observational Studies in Epidemiology guidelines. The study was conducted on a sample of 225 midwives working in the Polish public health service that consisted exclusively of women. The data was collected in 2 phases. The first phase took place from March to August 2018 and involved 133 midwives. The second phase took place during the COVID-19 pandemic, in February 2021, when a surge of coronavirus cases began in Poland. During this latter phase, data was collected from 92 midwives. Individuals surveyed in phases 1 and 2 came from the same institutions located in the following provinces: Lower Silesia, Mazovia, Opole, Podlasie, Pomerania, and Silesia. The sociodemographic and occupational characteristics of the participants are presented in Table 1.

Participation in the study was anonymous and voluntary, and it could be waived at any time. All participants were informed of the purpose of the study.
Table 1. Sociodemographic and occupational characteristics for Polish midwives working before (March-August 2018) and during (February 2021) COVID-19

\begin{tabular}{|c|c|c|}
\hline \multirow{2}{*}{ Variable } & \multicolumn{2}{|c|}{$\begin{array}{l}\text { Participants } \\
(\mathrm{N}=225)\end{array}$} \\
\hline & $\begin{array}{l}\text { before COVID-19 } \\
\qquad(\mathrm{N}=133)\end{array}$ & $\begin{array}{l}\text { during COVID-19 } \\
(\mathrm{N}=92)\end{array}$ \\
\hline Age [years] $(\mathrm{M} \pm \mathrm{SD})$ & $40.8 \pm 12.42$ & $43.07 \pm 12.32$ \\
\hline Seniority [years] $(\mathrm{M} \pm \mathrm{SD})$ & $17.44 \pm 12.47$ & $19.61 \pm 12.48$ \\
\hline Working time $[\mathrm{h} /$ week] $(\mathrm{M} \pm \mathrm{SD})$ & $46.26 \pm 16.53$ & $44.46 \pm 14.92$ \\
\hline \multicolumn{3}{|l|}{ Swift work [\%] } \\
\hline yes & 85.4 & 80.06 \\
\hline no & 14.6 & 19.04 \\
\hline \multicolumn{3}{|l|}{ Work places [\%] } \\
\hline 1 & 70.07 & 76.7 \\
\hline 2 & 24.8 & 19.8 \\
\hline$>2$ & 4.5 & 3.5 \\
\hline \multicolumn{3}{|l|}{ Ward of working [\%] } \\
\hline childbirth & 24.8 & 39.1 \\
\hline gyneacology & 15.8 & 6.5 \\
\hline maternity & 9.8 & 4.3 \\
\hline gyneacology-maternity & 21.8 & 23.9 \\
\hline neonatal & 5.3 & 4.3 \\
\hline obstetric and neonatal & 8.3 & 0 \\
\hline pragnancy pathology & 7.5 & 6.5 \\
\hline intensive therapy for newborns & 5.3 & 0 \\
\hline clinic & 1.5 & 2.2 \\
\hline oncology-gyneacology & 0 & 4.3 \\
\hline private practice & 0 & 8.7 \\
\hline \multicolumn{3}{|l|}{ Marital status [\%] } \\
\hline single & 11.6 & 9.7 \\
\hline informal relationship & 20.4 & 16.1 \\
\hline marriage & 62.8 & 67.7 \\
\hline widow & 0 & 0 \\
\hline divorced & 5.2 & 6.5 \\
\hline \multicolumn{3}{|l|}{ Education [\%] } \\
\hline midwifery high school & 8.3 & 10.9 \\
\hline bachelor's degree in midwifery & 84.2 & 47.9 \\
\hline master's degree in midwifery & 7.5 & 41.2 \\
\hline
\end{tabular}

In the first phase, the participants were recruited directly by researchers on hospital wards and through personal communication. Study questionnaires were left in the hospital wards and were collected by the project 
team after a set time. The second phase took place entirely remotely. Midwives were recruited through email, social media postings, and through personal communication. Participation in the study consisted of completing an encrypted online questionnaire. A formal requirement for participation in the study was to have worked in the midwifery profession for at least 1 year at the start of the first phase of research in 2018.

This study was approved by the Ethics Committee of the Institute of Psychology, University of Opole. The study was conducted in accordance with the guidelines of the Declaration of Helsinki, and all participants gave written informed consent. The collected data was stored and processed in a secure database.

\section{Data collection and research tools}

All participants completed a sociodemographic questionnaire and 3 psychological questionnaires as described below. All scales used in the study achieved sufficient reliability. Reliability indices were calculated for each group separately and are presented in tables along with the descriptive statistics.

\section{Sociodemographic questionnaire}

This questionnaire included questions about age, job tenure, work location/department, province, education, marital status, working hours, and shift work. In the second phase, the questionnaire was supplemented with questions about contact with infected persons and personal infection with SARS-CoV-2.

\section{Quantitative Workload Inventory}

Workload was measured using the Quantitative Workload Inventory (QWI) in a Polish adaptation [12]. The QWI is used to measure quantitative workload expressed as the effort required to complete a large number of tasks within a limited time frame. The questionnaire consists of 5 questions measuring 1 factor and includes questions such as: "How often are you forced to do more work than you are able to?" The responses are given on a 5-point Likert scale ranging from 1 (less than once a month or never) to 5 (several times a day). Thus, higher scores indicate an increased workload.

\section{Satisfaction With Job Scale}

Job satisfaction was measured using the Satisfaction With Job Scale (SWJS) [18]. This tool is used to measure the cognitive aspects of job satisfaction. The scale has a univariant structure and consists of 5 statements in the form of general assessments of the value of the work. A sample statement included in the tool is: "I have a great work environment." The answers are given on a 7-point scale, ranging from 1 (strongly disagree) to 7 (strongly agree). The level of job satisfaction can be inferred from the sum of the scores, with higher scores reflecting increased job satisfaction.

\section{Perceived Stress Scale}

The intensity of occupational stress was examined using the Perceived Stress Scale (PSS-10) in a Polish adaptation [19]. The scale consists of 10 positions and has a univariant structure. This questionnaire is designed to measure general occupational stress defined as an assessment of the perceived relationship between the individual and the work environment. Respondents assessed how often they felt an emotion or thought in a particular way over the past month as described in the question. The questionnaire contained questions such as: "During the past month, how often have you felt that you were not in control of important things in your professional life?" Each item on the test is rated on a 5-point scale. The different points of the scale are described as follows: 1 (never), 2 (almost never), 3 (sometimes), 4 (quite often), 5 (very often). The maximum score is $50 \mathrm{pts}$, and the minimum is $10 \mathrm{pts}$, and the higher the score, the greater the perceived stress at work. Questions 4, 5, 7, 8 had an inverted meaning.

\section{Statistical analyses}

The primary analytic approach was based on hierarchical regression analysis and mediation analysis, where occupational stress was the explanatory variable. The analyses were divided into 2 stages. In the first, hierarchical regression analysis was performed on the entire sample, and in the second, mediation analysis was performed for the 2 groups separately. In the regression model, the predictors used in the following steps were: 1) work before vs. during the pandemic, 2) infection with SARS-CoV-2, 3) workload, and 4) job satisfaction. The variables were entered into the regression model based on the assumptions of the JD-R model. First, the correlation between work requirements and occupational stress was examined. Next, a variable that is a general indicator of resources available at work (i.e., job satisfaction) was introduced into the tested model. In the mediation model, job satisfaction was the mediating variable. The model was tested separately on the 2 groups of midwives (i.e., those working before 
Table 2. Correlations between the study variables for Polish midwives working before (March-August 2018, N = 133) and during (February 2021, $\mathrm{N}=92$ ) COVID-19

\begin{tabular}{|c|c|c|c|c|c|c|}
\hline Variable & M & SD & \multicolumn{4}{|c|}{ Correlation } \\
\hline \multicolumn{7}{|l|}{ Before COVID-19 } \\
\hline 1. Seniority & 17.44 & 12.47 & & & & \\
\hline 2. Workload & 19.06 & 5.04 & -0.05 & 0.87 & & \\
\hline \multicolumn{7}{|l|}{ During COVID-19 } \\
\hline 1. Seniority & 19.61 & 12.48 & - & & & \\
\hline 2. Workload & 20.02 & 4.20 & -0.06 & 0.87 & & \\
\hline
\end{tabular}

Cronbach's $\alpha$ on the diagonal.

${ }^{*} \mathrm{p}<0.05,{ }^{* * *} \mathrm{p}<0.001$.

or during the COVID-19 pandemic). Only complete questionnaires were analyzed. Statistical analyses were performed using IBM SPSS version 21 software with the PROCESS add-on [20].

\section{RESULTS}

Analysis of intergroup differences revealed no significant differences in sociodemographic and formal workplace characteristics between midwives studied before and during the COVID-19 pandemic. In both groups, there was a positive correlation between age and job tenure $(r=0.96, p<0.001)$. In the group of midwives surveyed before the pandemic, the mean level of workload was at a medium-high level $(\mathrm{M}=19.06, \mathrm{SD}=5.04)$, and job satisfaction $(\mathrm{M}=21.63, \mathrm{SD}=6.07)$ and occupational stress $(\mathrm{M}=28.94, \mathrm{SD}=5.33)$ were moderate. In this group, job tenure $(\mathrm{r}=0.17, \mathrm{p}=0.045)$ and number of jobs $(r=0.18, p=0.032)$ significantly correlated with occupational stress. The correlation coefficients for the pre-pandemic study group are shown in Table 2. In the group of midwives working during the pandemic, the level of workload was also at a medium-high level $(M=20.02, S D=4.20)$, and the levels of job satisfaction $(M=22.20, S D=7.03)$ and job stress $(M=31.65$, $\mathrm{SD}=7.31)$ can be considered average. In the group working during the pandemic, shift work was positively correlated with workload $(r=0.39, \mathrm{p}=0.002)$ and negatively correlated with job satisfaction $(\mathrm{r}=-0.47, \mathrm{p}<0.001)$. The correlation coefficients for the study group working during the pandemic are shown in Table 2.
Among midwives surveyed during the pandemic, $77.5 \%$ had contact with a person infected with SARSCoV-2 in their primary workplace, $64.5 \%$ indicated having an immediate family member who had been infected with SARS-CoV-2, and $45.2 \%$ reported suffering from COVID-19 themselves.

The results of the hierarchical regression analysis indicated that performing work during the COVID-19 pandemic is a significant predictor of occupational stress among midwives, both when analyzed alone $(\beta=0.21, p=0.001)$ and when controlling for other variables $(\beta=0.13, p=0.017)$. Thus, the $\mathrm{H} 1$ hypothesis was supported. The results of an intergroup comparison analysis indicated significant differences between midwives studied before and during the pandemic in terms of the occupational stress experienced $(t=-3.21$, $\mathrm{p}=0.001, \mathrm{~d}=0.42$ ). Thus, hypothesis $\mathrm{H} 2$ was also supported. Personal infection with SARS-CoV-2 significantly increased the levels of occupational stress in midwives. The stress level was elevated by SARS-CoV-2 infection alone $(\beta=0.26, \mathrm{p}<0.001)$ and when controlling for other variables $(\beta=0.18, \mathrm{p}=0.002)$. This result supports hypothesis $\mathrm{H} 3$. When controlling for working during the pandemic and personal SARSCoV-2 infection, workload significantly increased occupational stress $(\beta=0.16, \mathrm{p}<0.001)$, while job satisfaction significantly decreased occupational stress ( $\beta=-0.49, \mathrm{p}<0.001)$. Thus, the $\mathrm{H} 4$ and $\mathrm{H} 5$ hypotheses were supported. The results of the subsequent hierarchical regression steps were statistically significant, and the included variables significantly increased 


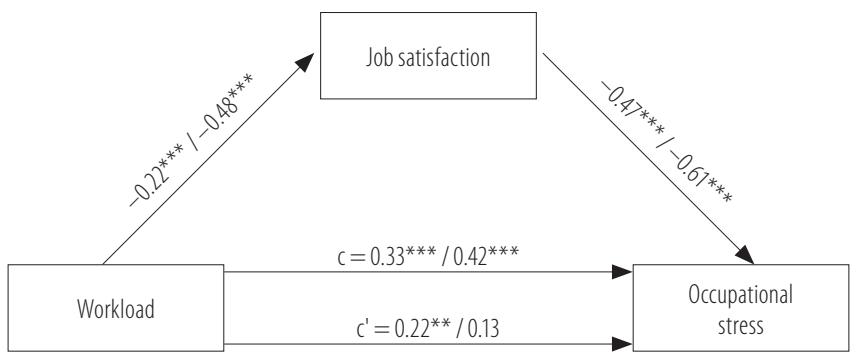

${ }^{* *} \mathrm{p}<0.01,{ }^{* * *} \mathrm{p}<0.001$.

Before slash - midwives working before COVID-19 pandemic $(\mathrm{N}=133), \mathrm{R}^{2}=0.32$, $\mathrm{F}(2,130)=31.19, \mathrm{p}<0.001$, after slash - midwives working during COVID-19 pandemic $(\mathrm{N}=92), \mathrm{R}^{2}=0.46, \mathrm{~F}(2,89)=39.15, \mathrm{p}<0.001$.

Figure 1. Results for mediation analysis

the percentage of explained variance in occupational stress. The detailed results of the regression analysis are shown in Table 3.

The results of the mediation analysis indicated that, in both groups of midwives, workload was negatively correlated with job satisfaction, and the association between workload and occupational stress was weaker when mediated by job satisfaction. The indirect effects in both groups were statistically significant. Job satisfaction mediated the correlation between workload and occupational stress both in the pre-pandemic group (indirect effect $\beta=0.10,95 \%$ CI: $0.021-0.206$ ) and in the pandemic group (indirect effect $\beta=0.29$, 95\% CI: 0.162-0.426). Thus, hypothesis H6 was supported.

Among midwives working before the pandemic, the negative associations between workload and job satisfaction $(\beta=-0.22, p=0.008)$, and between job satisfaction and occupational stress $(\beta=-0.47, \mathrm{p}<0.001)$, were weaker compared with midwives working during the pandemic $(\beta=-0.48, p<0.001$ and $\beta=-0.61$, $\mathrm{p}<0.001)$. In the pre-pandemic midwife group, the association between workload and stress $(\beta=0.33$, $\mathrm{p}<0.001)$ was weaker than in the group who worked during the pandemic $(\beta=0.42, \mathrm{p}<0.001)$. In addition, the study groups differed in the strength of the mediation effect. Among pre-pandemic midwives, mediation through job satisfaction reduced the negative effect of workload on stress, but the association between workload and stress remained significant $(\beta=0.22, \mathrm{p}=0.002)$. There was a total mediation effect in the group working during the pandemic. The inclusion of job satisfaction removed the significance of the correlation between

Table 3. Hierarchical regression results for occupational stress among Polish midwives $(\mathrm{N}=225)$ working before (March-August 2018) and during (February 2021) COVID-19

\begin{tabular}{|c|c|c|c|c|c|c|c|}
\hline Model & B & SE B & $95 \%$ CI & $\beta$ & $\mathrm{R}^{2}$ & $\Delta \mathrm{R}^{2}$ & $\mathrm{~F}(\mathrm{df})$ \\
\hline Model 1 & & & & & 0.04 & $0.04^{* *}$ & $10.35(1,223)$ \\
\hline constant & 26.22 & 1.25 & $23.74-28.70$ & & & & \\
\hline work during COVID-19 & 2.17 & 0.84 & $1.05-4.37$ & $0.21^{\star * *}$ & & & \\
\hline Model 2 & & & & & 0.09 & $0.05^{* * *}$ & $14.47(2,222)$ \\
\hline constant & 22.66 & 1.55 & $19.61-25.72$ & & & & \\
\hline work during COVID-19 & 1.15 & 0.91 & $-0.65-2.96$ & 0.09 & & & \\
\hline COVID-19 infection & 5.12 & 1.36 & $2.42-7.81$ & $0.26^{* * *}$ & & & \\
\hline Model 3 & & & & & 0.20 & $0.11^{\star * *}$ & $19.44(3,221)$ \\
\hline constant & 15.19 & 1.99 & $11.26-19.12$ & & & & \\
\hline work during COVID-19 & 0.98 & 0.86 & $-0.71-2.69$ & 0.07 & & & \\
\hline COVID-19 infection & 4.26 & 1.29 & $1.70-6.82$ & $0.22^{* * *}$ & & & \\
\hline workload & 0.44 & 0.08 & $0.28-0.60$ & $0.33^{* * *}$ & & & \\
\hline Model 4 & & & & & 0.44 & $0.23^{* * *}$ & $44.10(4,220)$ \\
\hline constant & 30.37 & 2.29 & $24.75-34.89$ & & & & \\
\hline work during COVID-19 & 1.72 & 0.73 & $0.29-3.16$ & $0.13^{*}$ & & & \\
\hline COVID-19 infection & 3.45 & 1.09 & $1.30-5.60$ & $0.18^{\star *}$ & & & \\
\hline workload & 0.22 & 0.07 & $0.08-0.36$ & $0.16^{* *}$ & & & \\
\hline job satisfaction & -0.50 & 0.05 & $-0.60-(-0.51)$ & $-0.49^{* * *}$ & & & \\
\hline
\end{tabular}

${ }^{*} \mathrm{p}<0.05,{ }^{* *} \mathrm{p}<0.01,{ }^{* * *} \mathrm{p}<0.001$ 
workload and occupational stress. Thus, hypothesis H7 was supported. The results of the mediation analysis are presented in Figure 1.

\section{DISCUSSION}

This is the first psychological study on the determinants of occupational stress in a group of Polish midwives working during the COVID-19 pandemic. The purpose of this study was to explore the correlations between workload, job satisfaction, and occupational stress and to compare the strength of these associations among midwives working in conditions before and during the COVID-19 pandemic. The study was conducted based on the JD-R model, and the results were consistent with the hypotheses of the model tested.

According to the JD-R model, job requirements become stressors when they entail a great deal of effort to maintain expected performance levels. The research presented here shows that job requirements can be related to the macro-social context. Working under the conditions of the COVID-19 pandemic is a significant predictor of occupational stress and the work is more stressful than working under pre-pandemic conditions. The level of stress experienced at work in the group of midwives studied during the pandemic is significantly higher, and the strength of the observed effect is moderate. Similar results were obtained by Krok and Zarzycka [21], indicating that pandemic risk is negatively correlated with the well-being of healthcare workers, and the strength of this correlation depends on coping strategies. This may mean that midwives use a maladaptive strategy of ruminating (negative deliberation) about the pandemic emergency and, therefore, they find the work more stressful. Previous research has supported the perseverative cognition hypothesis [8] in the context of well-being at work [7]. The results of the current research complement the state of knowledge about the correlations between the pandemic threat and occupational stress.

In hypothesis 3 it was proposed that a personal SARS$\mathrm{CoV}-2$ infection would be positively associated with occupational stress. It turns out that coronavirus infection significantly increases experienced occupational stress, with a higher regression coefficient on stress than working during the pandemic alone. Based on this observation, it can be extrapolated that ruminations about the pandemic are stressful but occupational stress levels are raised more significantly by real personal experience with the disease. According to the JD-R model, when employees are exposed to increased workload, they are more likely to use maladaptive coping strategies that exacerbate the stress [5]. The results of the current study suggest that the midwives who worked during the pandemic are psychologically burdened by the fear of infection, and that personal illness increased workplace stressors.

Previous studies have confirmed the positive association of workload with the psychological costs of work (i.e., work-related burnout, depressive symptoms, and physical fatigue), but did not include a group of Polish midwives [22], and did not consider job satisfaction as a mediator. The level of workload in the study sample was medium-high, which may be the basis for the conclusion that Polish midwives experience high mental and physical effort at work. Studies have shown that workload understood quantitatively (e.g., many responsibilities, little time) significantly increases the level of occupational stress.

Hypotheses 5 and 6 proposed that job satisfaction was significantly associated with occupational stress, and that the correlation between workload and stress in a group of midwives was mediated by job satisfaction. The results of the current study are part of a trend documenting the importance of job satisfaction in coping with stress in the medical profession. Previous studies in nurses, based on the JD-R model, have confirmed a strong negative correlation between job satisfaction, increased requirements at work, and occupational stress, leading to work-related burnout [23]. Job satisfaction is strongly influenced by working conditions, which, in the context of the current results, suggests that the midwives studied here rated the social and organizational resources at work as sufficient to cope with occupational stress. At the same time, it should be noted that job satisfaction in this study sample was at a moderate level. Nevertheless, job satisfaction significantly and strongly reduced occupational stress, both directly, and as a mediator of the workload-occupational stress correlation.

Other research has also pointed to the mediating role of job satisfaction in the correlation between occupational stress and work-related burnout [24]. In this project, the emphasis of the investigation was placed on the determinants of stress. The positive influence of job satisfaction on coping with stress is particularly evident among midwives working during the COVID-19 pandemic. This may imply that midwives subjected to the stress of working during a pandemic use their resources more intensively to cope with occupational stress. It appears that the pandemic context reinforces 
the role of positive cognitive appraisal of working conditions in reducing work-related stress.

Our results indicate that workload and job satisfaction explain a greater percentage of the variance in occupational stress in the group working during the pandemic compared those working in the period before the pandemic. That is, a pandemic situation increases the importance of requirements and resources at work, compared to the factors shaping occupational stress located outside the workplace. This finding is in agreement with previous studies based on the JD-R model, which showed that employees are particularly sensitive to working conditions [25]. Our results suggest that $20 \%$ of the variance in occupational stress of midwives working during the pandemic is due to perceived COVID-19 risk and workload (job requirements), and $23 \%$ is determined by job satisfaction. These results show that the negative correlation between job satisfaction and occupational stress is stronger in difficult situations. The context of the pandemic reinforces the role of job satisfaction in coping with workloads and occupational stress.

\section{Limitations}

A limitation of the research presented here is the use of self-report measures. Participants in the study, especially those in the group studied during the pandemic, may have given socially expected responses (i.e., indicating that their jobs were highly stressful). All data on the independent and dependent variables were collected by questionnaire method, which exposes the results to this common method error. An additional limitation of the current study is the cross-sectional nature of the research. The lack of a longitudinal approach limits the accuracy in inferring causal relationships. Midwives from the same facilities participated in both phases of the study, but a longitudinal design was not possible due to the pandemic. However, controlling for the workplace variable and the lack of significant differences in sociodemographic variables suggests that the same midwives participated in both phases of the study. The value of the results is also affected by limiting the stress predictors to job requirements and job satisfaction. Previous data support both the direct and indirect role of resources at work in coping with stress in midwives [26]. In this study, the resources were omitted for several reasons. First, the current social context is more conducive to an increase in work requirements than resources for health professionals [27]. In addition, work requirements have a stronger impact on shaping the psychological costs of work, an observation supported by previous JD-Rbased studies [25]. Another limitation is the sample size. The study involved 225 midwives working in 6 of Poland's 16 provinces. This, along with the aforementioned limitations, narrow the generalizability of the results. On the other hand, research on the psychological determinants of Polish midwives' stress, especially in the context of the COVID-19 pandemic, is, to the authors' knowledge, scarce.

\section{Practical implications}

The current study has a practical dimension as it draws attention to a professional group that has been largely neglected in the Polish psychological literature. Midwives are responsible for maternal and newborn health outcomes. Maternity care in a pandemic situation takes on special moral significance. Women who are pregnant and giving birth, despite the difficult conditions, expect professional and safe healthcare. The results of this study indicate that working during a pandemic is positively associated with stress levels, with the midwives surveyed experiencing medium-high levels of workload. Factors that may attenuate the stressful effects of the pandemic include job autonomy, flexible work schedules, and sufficient material resources. These factors reduce stress, and improve sleep quality [28] work-life balance, productivity, performance, and well-being at work [29]. The current findings also draw attention to the importance of stress prevention in the healthcare sector. Above all, the level of quantitative workload needs to be lowered. This could translate into higher levels of job satisfaction and lower levels of stress, ultimately saving midwives from experiencing work-related burnout. Results from previous studies indicate that work-related burnout is negatively associated with healthcare quality. Patients treated by overworked and exhausted staff report lower levels of safety in relation to their stay in the healthcare facility, and highly stressed staff make more errors at work [30].

\section{CONCLUSIONS}

This study adds to the literature on the correlations between workload, job satisfaction, and occupational stress in Polish midwives working before and during the COVID-19 pandemic. The results indicate that working during the pandemic, personal infection with coronavirus, and workload significantly increases occupational stress. In the group of midwives working during the pandemic, the level of experienced occupational 
stress was significantly higher compared to those working before the pandemic. Job satisfaction strongly decreases midwives' levels of experienced occupational stress both directly and as a mediator of the workload-occupational stress relationship. The obtained results are important because they address the problem of occupational stress during the COVID-19 pandemic in a group critically important to the healthcare sector. To the best of the authors' knowledge, it is the first study on this topic concerning Polish midwives.

\section{REFERENCES}

1. Ministerstwo Zdrowia RP. Raport zakażeń koronawirusem (SARS-CoV-2) [SARS-CoV-2 in Poland - Report]. Ministerstwo Zdrowia RP 2021 [cited 20 May 2021]. Available from: https://www.gov.pl/web/koronawirus/wy kaz-zarazen-koronawirusem-sars-cov-2.

2. Maciaszek J, Ciulkowicz M, Misiak B, Szczesniak D, Luc D, Wieczorek T, et al. Mental Health of Medical and NonMedical Professionals during the Peak of the COVID-19 Pandemic: A Cross-Sectional Nationwide Study. J Clin Med. 2020;9(8):2527. https://doi.org/10.3390/jcm9082527.

3. Rodríguez Coll P, Casañas R, Collado Palomares A, Maldonado Aubián G, Duran Muñoz F, Espada-Trespalacios X, et al. Women's childbirth satisfaction and obstetric outcomes comparison between two birth hospitals in Barcelona with different level of assistance and complexity. Cent Eur J Nurs Midw. 2021;12(1):235-44. https://doi. org/10.15452/cejnm.2020.11.0038.

4. Aksoy YE, Koçak V. Psychological effects of nurses and midwives due to COVID-19 outbreak: The case of Turkey. Arch Psychiatr Nurs. 2020;34(5):427-33. https://doi.org/ 10.1016/j.apnu.2020.07.011.

5. Bakker AB, de Vries JD. Job Demands-Resources theory and self-regulation: new explanations and remedies for job burnout. Anxiety Stress Coping. 2021;34(1):1-21. https:// doi.org/10.1080/10615806.2020.1797695.

6. Arslan G, Yildırım M. Coronavirus stress, meaningful living, optimism, and depressive symptoms: a study of moderated mediation model. Aust J Psychol. 2021;73(2):113124. https://doi.org/10.1080/00049530.2021.1882273.

7. Bakker AB, van Wingerden J. Rumination about COVID-19 and employee well-being: The role of playful work design. Can Psychol. 2021;22(1):73-79. https://doi.org/10.1037/cap 0000262.

8. Brosschot JF, Gerin W, Thayer JF. The perseverative cognition hypothesis: A review of worry, prolonged stress-related physiological activation, and health. J Psychosom Res. 2006;60(2): 113-24. https://doi.org/10.1016/j.jpsychores.2005.06.074.
9. Baumann S, Gaucher L, Bourgueil Y, Saint-Lary O, Gautier S, Rousseau A. Adaptation of independent midwives to the COVID-19 pandemic: A national descriptive survey. Midwifery. 2021;94:102918. https://doi.org/10.1016/ j.midw.2020.102918.

10. Kniffin KM, Narayanan J, Anseel F, Antonakis J, Ashford SP, Bakker AB, et al. COVID-19 and the workplace: Implications, issues, and insights for future research and action. Am Psychol. 2021;76(1):63-77. https://doi.org/10. 1037/amp0000716.

11. Salehi M, Amanat M, Mohammadi M, Salmanian M, Rezaei N, Saghazadeh A, et al. The prevalence of post-traumatic stress disorder related symptoms in Coronavirus outbreaks: A systematic-review and meta-analysis. J Affect Disord. 2021;282:527-38. https://doi.org/10.1016/ j.jad.2020.12.188.

12. Baka $€$, Bazińska R. Polish adaptation of three self-report measures of job stressors: the Interpersonal Conflict at Work Scale, the Quantitative Workload Inventory and the Organizational Constraints Scale. Int J Occup Saf Ergon. 2016;22(1):32-9. https://doi.org/10.1080/1080354 8.2015.1116816.

13. Balducci C, Alessandri G, Zaniboni S, Avanzi L, Borgogni L, Fraccaroli F. The impact of workaholism on day-level workload and emotional exhaustion, and on longer-term job performance. Work Stress. 2021;35(1):626. https://doi.org/10.1080/02678373.2020.1735569.

14. Scanlan JN, Still M. Relationships between burnout, turnover intention, job satisfaction, job demands and job resources for mental health personnel in an Australian mental health service. BMC Health Serv Res. 2019;19(1):62. https://doi.org/10.1186/s12913-018-3841-z.

15. Kessler SR, Lucianetti L, Pindek S, Zhu Z, Spector PE. Job satisfaction and firm performance: Can employees' job satisfaction change the trajectory of a firm's performance? J Appl Soc Psychol. 2020;50(10):563-72. https:// doi.org/10.1111/jasp.12695.

16. Akyurt N. Job satisfaction and perceived stress among radiology technicians: a questionnaire survey in relation to sociodemographic and occupational risk factors. Int Arch Occup Environ Health. Forthcoming 2021.

17. Lapointe-Shaw L, Bouck Z, Howell NA, Lange T, Orchanian-Cheff A, Austin PC, et al. Mediation analysis with a time-to-event outcome: a review of use and reporting in healthcare research. BMC Med Res Methodol. 2018;18(1):118. https://doi.org/10.1186/s12874-0180578-7.

18. Zalewska AM. Skala satysfakcji z pracy - pomiar poznawczego aspektu ogólnego zadowolenia $\mathrm{z}$ pracy [The Satisfaction With Job Scale - measurement of cognitive 
aspect of overall job satisfaction]. Acta Universitatis Lodziensis Folia Psychologica. 2003;7:49-61.

19. Chirkowska-Smolak T, Grobelny J. Konstrukcja i wstępna analiza psychometryczna Kwestionariusza Postrzeganego Stresu w Pracy (PSwP) [The design and preliminary psychometric analysis of The Perceived Stress at Work Questionnaire]. Czasopismo Psychologiczne. 2016;22(1): 131-9.

20. Hayes AF. Introduction to Mediation, Moderation, and Conditional Process Analysis. New York, NY: Guilford Press; 2017.

21. Krok D, Zarzycka B. Risk Perception of COVID-19, Meaning-Based Resources and Psychological Well-Being amongst Healthcare Personnel: The Mediating Role of Coping. J Clin Med. 2020;9(10). https://doi.org/10.3390/ jcm9103225.

22. Baka Ł. Does job burnout mediate negative effects of job demands on mental and physical health in a group of teachers? Testing the energetic process of Job DemandsResources model. Int J Occup Med Environ Health. 2015; 28(2):335-46. https://doi.org/10.13075/ijomeh.1896.00246.

23. Kaiser S, Patras J, Adolfsen F, Richardsen AM, Martinussen M. Using the Job Demands-Resources Model to Evaluate Work-Related Outcomes Among Norwegian Health Care Workers. Sage Open. 2020;10(3):2158244020 947436. https://doi.org/10.1177/2158244020947436.

24. Wu F, Ren Z, Wang Q, He M, Xiong W, Ma G, et al. The relationship between job stress and job burnout: the mediating effects of perceived social support and job satisfaction. Psychol Health Med. 2021;26(2):204-11, https://doi.org/10.1080/13548506.2020.1778750.

25. Hakanen JJ, Bakker AB, Schaufeli WB. Burnout and work engagement among teachers. J Sch Psychol. 2006;43(6): 495-513. https://doi.org/10.1016/j.jsp.2005.11.001.

26. Jasiński AM. Perinatal stress events and burnout among midwives in Poland. The mediating role of self-efficacy. Cent Eur J Nurs Midw. 2021;12(1):267-78. https://doi. org/10.15452/cejnm.2021.12.0002.

27. Duarte I, Teixeira A, Castro L, Marina S, Ribeiro C, Jácome C, et al. Burnout among Portuguese healthcare workers during the COVID-19 pandemic. BMC Public Health. 2020;20(1):1885. https://doi.org/10.1186/s12889020-09980-z.

28. Magnavita N, Tripepi G, Di Prinzio RR. Symptoms in Health Care Workers during the COVID-19 Epidemic. A Cross-Sectional Survey. Int J Environ Res Public Health 2020;17(14):5218. https://doi.org/10.3390/ijerph17145218.

29. Jamal MT, Anwar I, Khan NA, Saleem I. Work during COVID-19: assessing the influence of job demands and resources on practical and psychological outcomes for employees. Asia-Pacific Journal of Business Administration. Forthcoming 2021.

30. Sováriová Soósová M. Association between nurses' burnout, hospital patient safety climate and quality of nursing care. Cent Eur J Nurs Midw 2021;12(1):245-56. https:// doi.org/10.15452/cejnm.2020.11.0039.

This work is available in Open Access model and licensed under a Creative Commons Attribution-NonCommercial 3.0 Poland License - http://creativecommons.org/licenses/by-nc/3.0/pl/deed.en. 